\title{
Patient Perspectives on Biologics for Axial Spondyloarthritis in a Cross-sectional Study in a Predominantly Female Population: Treatment Satisfaction, Wear-off Between Doses, and Use of Supplemental Medication
}

\author{
W. Benjamin Nowell (D) - Kelly Gavigan • Theresa Hunter • Rebecca J. Bolce · Jeffrey R. Lisse • \\ Carol Himelein · Suchita Dubey · Jeffrey R. Curtis · Jessica A. Walsh
}

Received: September 17, 2021 / Accepted: December 13, 2021 / Published online: December 27, 2021

(C) The Author(s) 2021

\begin{abstract}
Introduction: There is limited information regarding treatment experience of patients with axial spondyloarthritis/ankylosing spondylitis (axSpA/AS) receiving biological disease-modifying antirheumatic drugs (bDMARDs). Here we characterize patient experiences and perspectives, including satisfaction among those currently treated with bDMARD therapy for axSpA/ AS. We also assess the use of supplemental medication during perceived wear-off between doses.
\end{abstract}

Methods: Adult participants from the United States within the ArthritisPower registry with

Supplementary Information The online version contains supplementary material available at https:// doi.org/10.1007/s40744-021-00417-6.

W. B. Nowell $(\varangle) \cdot$ K. Gavigan

Global Healthy Living Foundation, Upper Nyack, NY, USA

e-mail: bnowell@ghlf.org

T. Hunter · R. J. Bolce · J. R. Lisse - C. Himelein .

S. Dubey

Eli Lilly and Company, Indianapolis, IN, USA

J. R. Curtis

The University of Alabama at Birmingham,

Birmingham, AL, USA

\section{J. A. Walsh}

George E. Wahlen Department of Veterans Affairs Medical Center, The University of Utah, Salt Lake City, UT, USA physician-diagnosed axSpA/AS were invited to complete electronic patient-reported outcome measures and an online survey about their perspectives of treatment. Analysis compared patient characteristics and treatment satisfaction by whether wear-off in axSpA/AS between bDMARD doses was reported.

Results: Of 128 patients currently taking a DMARD, the mean age was 46.9 (10.3) years, $82.0 \%$ were female, and $93.8 \%$ were White. A total of 78 (60.9\%) perceived wear-off with their current bDMARD before the next dose, 19 $(14.8 \%)$ did not experience wear-off and 31 (24.2\%) were unsure about wear-off. Mean (standard deviation [SD]) Bath Ankylosing Spondylitis Disease Activity Index (BASDAI) score indicated poor disease control in all patients receiving bDMARDs (6.4 [1.8]); worse for those perceiving wear-off between doses versus those who did not perceive wear-off or were unsure (6.8 [1.6] vs. 5.9 [2.0], $p=0.011$ ). Patients experiencing wear-off reported being 'very satisfied' or 'somewhat satisfied' with their treatment less frequently than patients without wear-off (73.1 vs. $89.5 \%$, respectively). Of patients reporting wear-off, $82.1 \%(n=64)$ used supplemental medications during wear-off (non-steroidal anti-inflammatory drugs [68.8\%, $n=44]$, muscle relaxants [42.2\%, $n=27]$, and/ or opioids [37.5\%, $n=24]$ ).

Conclusions: In a predominantly female sample of bDMARD-treated patients with axSpA/AS and high disease activity, the majority 
expressed treatment satisfaction. However, most experienced wear-off between doses and relied on supplemental medications, including opioids, to manage symptoms.

Keywords: Axial spondyloarthritis; Ankylosing spondylitis; Biologics; Efficacy; Satisfaction; Waning; Wear-off

\section{Key Summary Points}

Why carry out this study?

The literature about wear-off between doses and other reasons for declining efficacy of biologics in patients with axial spondyloarthritis (axSpA) or ankylosing spondylitis (AS) is limited.

In this study, we characterized patient experiences and perspectives with biological disease-modifying antirheumatic drug (bDMARD) treatments for axSpA/AS, including satisfaction, and use of supplemental treatments when patients experienced symptoms of wear-off between doses in a predominantly female population.

\section{What was learned from the study?}

In a predominantly female sample of bDMARD-treated patients with axSpA/AS and high disease activity, the majority expressed treatment satisfaction.

Most patients experienced wear-off between doses and relied on supplemental medications, including opioids, to manage symptoms.

\section{INTRODUCTION}

Axial spondyloarthritis (axSpA), comprising ankylosing spondylitis (AS) and non-radiographic axSpA (nr-axSpA), is a chronic inflammatory rheumatic disease that affects the sacroiliac joints and spine. AxSpA/AS commonly begins in patients aged $<40$ years. Inflammatory back pain is a common manifestation of the disease [1-3]. In the United States, the population prevalence of axSpA ranges from 0.9 to $1.4 \%$ [4].

The American College of Rheumatology/ Spondylitis Association of America/Spondyloarthritis Research and Treatment Network Recommendation 2019 updates suggest that the goals of axSpA/AS treatment are to alleviate symptoms, improve functioning, maintain work ability, reduce disease complications, and minimize skeletal damage [5]. However, patients and physicians often have different perspectives on the management of axSpA/AS. While physicians focus more on pain and stiffness, patients may also equally prioritize impact on work, friends, and family [6, 7]. To optimize therapy in axSpA, it is important to recognize individual needs of patients [5].

Biological disease-modifying antirheumatic drugs (bDMARDs) are known to improve signs and symptoms in patients with axSpA/AS [8-12]. Remission or low disease activity are the main treatment targets for the disease and indicators of the effectiveness of bDMARDs [13]. To achieve these desired outcomes and maintain disease control, bDMARD treatment is often continued indefinitely in clinical practice [14]. In addition, despite the appropriate selection, dosing, and adherence of bDMARDs, many patients with axSpA/AS discuss changing their biologics with their physicians [15]. One of the challenges with biological treatment is 'wear-off' between doses, reflecting the experience of diminished treatment efficacy [16]. Patients with axSpA/AS may experience wear-off as worsening of symptoms prior to each dose, which could result in patients using supplemental medications to manage signs and symptoms or requesting changes in their medication.

The literature regarding wear-off between doses and other reasons for declining efficacy of biologics in patients with axSpA/AS is limited. In this study, we aimed to characterize patient experiences and perspectives with bDMARD treatments for axSpA/AS, including satisfaction, and use of supplemental treatments when 
patients experience symptoms of wear-off between doses.

\section{METHODS}

\section{Study Design and Participants}

This was a non-site based, cross-sectional primary data collection study. Study participants were recruited from the ArthritisPower registry, which is a collaboration between the non-profit Global Healthy Living Foundation and rheumatology researchers at the University of Alabama, Birmingham, USA. Launched in 2015, ArthritisPower comprises members with a selfreported rheumatic and musculoskeletal disease diagnosis who have provided consent to participate in research studies and provide data via the ArthritisPower app using a smartphone or web-based equivalent [17]; the registry's participants governing board approved the study [18].

Participants eligible to enter the study included members of the ArthritisPower registry who were residents of the USA or Puerto Rico $\geq 19$ years of age ( $\geq 21$ for Puerto Rico residents) with a self-reported physician diagnosis of AS or axSpA (as indicated by survey screening questions), and with access to a computer or smartphone to complete online surveys. Participants were sent unique survey links via e-mail invitation reading, "Understanding AS: What Is Your Experience with axSpA/AS?" and specifying that the goal of the study was to understand patients' perspectives on axSpA/AS symptoms and quality of life, and their treatment decision-making process. Up to two e-mail reminders were sent to non-responders. A total of 2727 eligible members of ArthritisPower received invitations to participate in the study. E-mails were opened by $48 \%$ $(1321 / 2727)$ of those eligible and the registration link was clicked by $29 \%$ of those who saw it (387/1321).

After consent was obtained, participants were asked to complete the survey screening questions and were then directed to the ArthritisPower app where they responded to five electronic patient-reported outcome (ePRO) assessments, followed by an 81-item online survey developed in partnership with patient research partners. The survey collected data on current disease activity via the Bath Ankylosing Spondylitis Disease Activity Index (BASDAI), which is scored on a $0-10$ scale with score $\geq 4$ indicating suboptimal control of disease [19]. The survey also included instruments developed by the National Institutes of Health for the Patient-Reported Outcomes Measurement Information System (PROMIS): PROMIS Pain Interference-computerized adaptive testing (CAT) version, PROMIS Physical Function-CAT, PROMIS Sleep Disturbance-CAT, and two oneitem questions adapted from the PROMIS Global Health measure: physical health and depression [20]. PROMIS short forms are considered reliable measures of disease activity in axSpA/AS, where higher PROMIS physical function score indicates better physical function, whereas higher PROMIS sleep disturbance or pain interference scores indicate worse outcome [21].

Information about the participants' symptom history, family history, medications, and their perceptions about biologics were captured. Those participants who reported ever being treated with a bDMARD were asked questions about their experience on such treatment and whether they perceived wear-off between doses. The survey was programmed using Health Insurance Portability and Accountability Actcompliant SurveyMonkey software and took participants 20-35 min to complete. The study was reviewed and deemed exempt by Advarra Institutional Review Board (Advarra IRB protocol \#00039559).

\section{Statistical Analysis}

Descriptive summary statistics of participant demographics were conducted for the overall cohort and by treatment subgroup based on whether a participant was currently on a bDMARD or not, and on the level of satisfaction with the current treatment. Categorical variables were analyzed by frequency counts and percentages. Continuous variables were analyzed by mean (standard deviation [SD]), minimum and maximum. Chi-square and analysis of 
variance (ANOVA) tests were used to determine significant differences in demographic characteristics. Chi-square tests were used to determine significant differences in treatment satisfaction by bDMARD wear-off (yes vs. no) and significance was determined at an alpha of 0.05. Data were analyzed as observed with no imputation for missing data. Binary logistic regression analysis was conducted to assess the factors associated with bDMARD wear-off. All analyses were done using SAS version 9.4.

\section{RESULTS}

\section{Participants and Demographic Characteristics}

A total of 353 members completed the screener of whom 128 participants were on bDMARD treatment, met the inclusion criteria, and completed the ePROs and survey. Overall, the mean (SD) age of 128 participants was 46.9 (10.3) years, $82.0 \%$ were female, and 93.8\% were White. Of 128 participants who used any bDMARD for axSpA/AS, 78 (60.9\%) reported that their current biologic wears off before the next dose, 19 (14.8\%) did not report a wear-off, and $31(24.2 \%)$ were unsure of their answer. The mean (SD) age of the subgroup of 78 participants who reported wear-off between bDMARD doses was 46.1 (9.2) vs. 48.2 (11.8) for 50 participants who did not perceive a wear-off between bDMARD doses or were not sure of the effect $(p=0.247)$.

Of 78 participants who experienced a wearoff between bDMARD doses, 69 (88.5\%) were female; whereas 37 (74.0\%) of the remaining 50 participants were female. Other demographic characteristics by wear-off between bDMARD doses are presented in Table 1 . All 128 participants receiving bDMARDs reported being diagnosed by a physician with a form of spondyloarthritis. Of those, 111 (86.7\%) reported a diagnosis of AS and $11(8.6 \%)$ reported a diagnosis of axSpA by a rheumatologist. On average, these participants experienced a gap of 11 years from when they reported first noticing axSpA symptoms (mean [SD] age 26.9 [12.2]) and receiving an initial axSpA (or AS) diagnosis by a physician (mean [SD] age 37.9 [12.1]); the majority ( $n=69 ; 53.9 \%)$ of patients were HLAB27 positive (Table 1). Among 78 participants who experienced wear-off, a significantly greater number of participants (37 [47.4\%]) used conventional synthetic DMARDs (e.g., methotrexate, sulfasalazine) as a concurrent medication versus 18 (36.0\%) participants who did not experience wear-off or were unsure of their answer $(p=0.202)$. The participants who experienced wear-off between doses had worse mean (SD) BASDAI and PROMIS sleep disturbance scores versus those who did not or were unsure (BASDAI: 6.8 [1.6] vs. 5.9 [2.0]: $p=0.011$; PROMIS Sleep Disturbance: 61.2 [7.7] vs. $57.6[9.3]: p=0.017$ ) (Table 1 ). We also conducted a logistic regression analysis to assess the variables associated with bDMARD wear-off (Supplementary Table S1). The variables for improvement in axSpA symptoms (axSpASymptomsImp), age of symptom onset (AgeSymptomsStart), and no positive HLA-B27 test (noHLA) were statistically significant ( $p=0.0161,0.0284$, and 0.0195, respectively), and being female, although positively correlated with DMARD wear-off, was not statistically significant $(p=0.0660)$.

\section{Treatment Satisfaction by Wear-Off Between Doses of Biologics}

Among all 78 participants who experienced wear-off, $13(16.7 \%)$ were 'very satisfied', 44 (56.4\%) were 'somewhat satisfied', 16 (20.5\%) were 'somewhat dissatisfied', and 5 (6.4\%) were 'very dissatisfied' with their current axSpA treatment (Fig. 1). Out of 19 participants who did not experience wear-off, 0 were 'very dissatisfied', 9 (47.4\%) were 'somewhat satisfied', 8 (42.1\%) were 'very satisfied', 2 $(10.5 \%)$ were 'somewhat dissatisfied' (Fig. 1). In both of the groups, the majority of the patients were either 'very satisfied' or 'somewhat satisfied'. However, in the group that did not experience wear-off, more than 90\% patients were either 'very satisfied' or 'somewhat satisfied' ( $p=0.077)$. 
Table 1 Demographic and clinical characteristics by wear-off between bDMARD doses $(N=128)$

\begin{tabular}{|c|c|c|c|c|}
\hline & \multicolumn{4}{|c|}{ Mean $( \pm$ SD) or $n(\%)$} \\
\hline & $\begin{array}{l}\text { Pts currently on } \\
\text { bDMARD } \\
(N=128)\end{array}$ & $\begin{array}{l}\text { Wear-off between } \\
\text { bDMARD doses } \\
(N=78)\end{array}$ & $\begin{array}{l}\text { No wear-off/ } \\
\text { not sure } \\
(N=50)\end{array}$ & $p$ value \\
\hline Age & $46.9(10.3)$ & $46.1(9.2)$ & $48.2(11.8)$ & 0.247 \\
\hline Female & $106(82.8)$ & $69(88.5)$ & $37(74.0)$ & $0.034^{*}$ \\
\hline White & $115(89.8)$ & $70(89.7)$ & $45(90.0)$ & 0.963 \\
\hline Body mass index & $30.9 \pm 7.8$ & $31.2 \pm 8.5$ & $30.4 \pm 6.6$ & 0.575 \\
\hline \multicolumn{5}{|l|}{ Addition of current medications to bDMARD } \\
\hline $\begin{array}{l}\text { Conventional synthetic DMARD (e.g., } \\
\text { methotrexate, sulfasalazine) }\end{array}$ & $55(43.0)$ & $37(47.4)$ & $18(36.0)$ & 0.202 \\
\hline Prescription NSAID & $59(46.1)$ & $39(50.0)$ & $20(40.0)$ & 0.268 \\
\hline Other prescription medication $^{a}$ & $89(69.5)$ & $56(71.8)$ & $33(66.0)$ & 0.487 \\
\hline $\begin{array}{l}\text { Noticed improvement in symptoms related to } \\
\text { axSpA since starting current bDMARD }\end{array}$ & $80(62.5)$ & $51(65.4)$ & $29(58.0)$ & 0.400 \\
\hline $\begin{array}{l}\text { Noticed improvement in symptoms NOT } \\
\text { related to axSpA since starting current } \\
\text { bDMARD }\end{array}$ & $40(31.3)$ & $22(28.2)$ & $18(36.0)$ & 0.353 \\
\hline Age first noticed symptom of axSpA & $26.9(12.2)$ & $24.1(11.4)$ & $31.3(12.2)$ & $0.001^{*}$ \\
\hline Age at diagnosis of axSpA & $37.9(12.1)$ & $36.8(12.2)$ & $39.6(11.8)$ & 0.197 \\
\hline Diagnosis of AS by physician & $111(86.7)$ & $69(88.5)$ & $42(84.0)$ & 0.468 \\
\hline Diagnosis of axSpA by physician & $11(8.6)$ & $7(9.0)$ & $4(8.0)$ & 0.848 \\
\hline Ever received positive HLA-B27 test & $69(53.9)$ & $38(48.7)$ & $31(62.0)$ & 0.141 \\
\hline BASDAI $^{\mathrm{b}}$ & $6.4 \pm 1.8$ & $6.8 \pm 1.6$ & $5.9 \pm 2.0$ & $0.011^{*}$ \\
\hline PROMIS pain interference ${ }^{c}$ & $65.3 \pm 5.7$ & $66.0 \pm 5.1$ & $64.3 \pm 6.4$ & 0.094 \\
\hline PROMIS physical function ${ }^{c}$ & $36.7 \pm 5.6$ & $36.1 \pm 5.3$ & $37.7 \pm 5.8$ & 0.108 \\
\hline PROMIS sleep disturbance ${ }^{c}$ & $59.8 \pm 8.5$ & $61.2 \pm 7.7$ & $57.6 \pm 9.3$ & $0.017^{*}$ \\
\hline
\end{tabular}

$A S$ ankylosing spondylitis, axSpA axial spondyloarthritis, BASDAI Bath Ankylosing Spondylitis Disease Activity Index, bDMARD biological disease-modifying antirheumatic drugs, NSAID non-steroidal anti-inflammatory drugs, PROMIS Patient-Reported Outcomes Measurement Information System, SD standard deviation

* Statistical significance between groups of patients who experienced wear-off between bDMARD doses and those who experienced no wear-off or were not sure, $p<0.05$

${ }^{a}$ Other prescription medications: muscle relaxers, nerve pain medications or anti-depressants, and opioids

${ }^{b}$ BASDAI is scored on a $0-10$ scale with score $\geq 4$ indicating suboptimal control of disease

${ }^{c}$ PROMIS measures use T-score metric in which 50 is mean, 10 is standard deviation (SD) of US population; higher T-score $=$ more of concept measured; higher PROMIS physical function scores indicate better physical function, whereas higher PROMIS sleep disturbance or pain interference scores indicate worse outcome 


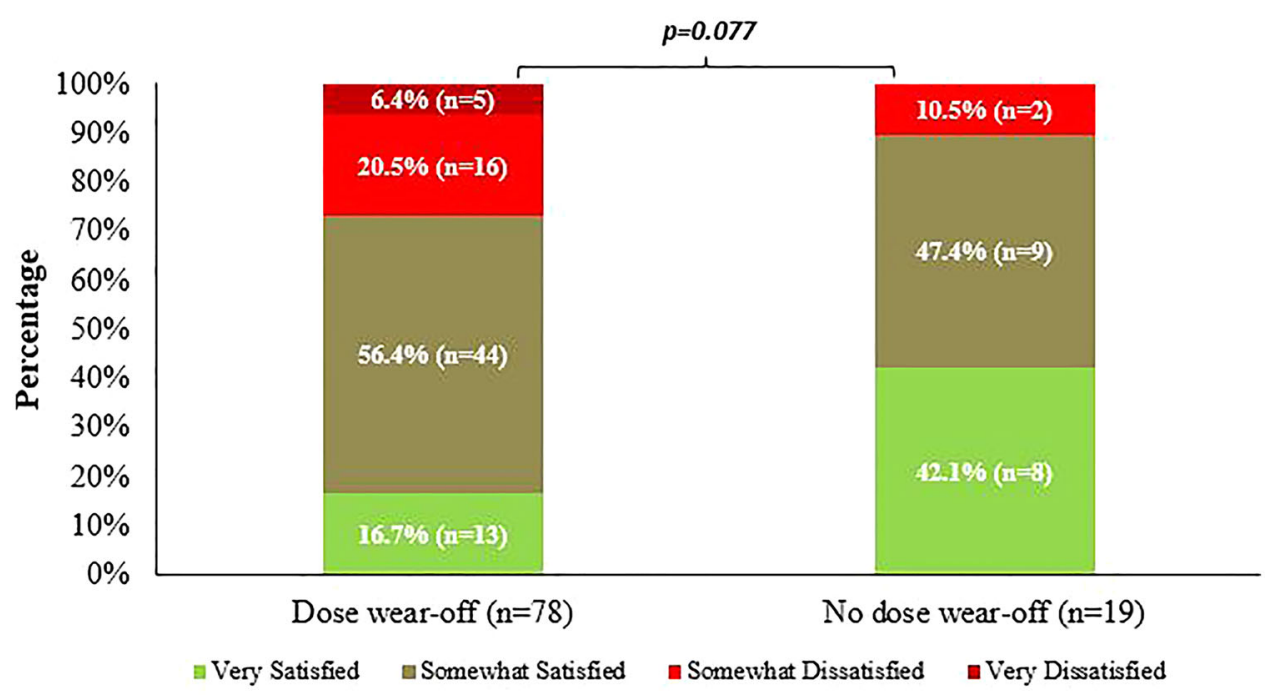

Fig. 1 Patient satisfaction with axSpA treatment by wearoff between bDMARD doses. No statistical significance between groups of patients who experienced wear-off between bDMARD doses and those who experienced no

wear-off or were not sure, $p<0.05$. axS $p A$ axial spondyloarthritis, $b D M A R D$ biological disease-modifying antirheumatic drugs

\section{bDMARDs Currently Taken by Patients}

Of the 128 participants receiving bDMARDs (adalimumab [n=40]; infliximab $[n=18]$; etanercept $[n=16]$; certolizumab $[n=14]$; golimumab [ $n=12]$; secukinumab $[n=28]$ ), the majority reported wear-off between doses (adalimumab [58\%]; infliximab [83\%]; etanercept [50\%]; certolizumab [50\%]; golimumab [83\%]; secukinumab [57\%]) (Fig. 2). The proportion of participants with the order in which they received bDMARDs is presented in Supplementary Table S1. A total of $62.5 \%$ (25 out of 40) participants received adalimumab as their first bDMARD.

\section{Additional Medications or Supplements Used When bDMARD Wear-Off is Perceived}

Of 78 participants who experienced wear-off between bDMARD doses, 64 participants used additional medications and supplements to mitigate the symptoms. A total of $44(68.8 \%)$ participants used non-steroidal anti-inflammatory drugs (NSAIDs), 27 (42.2\%) used muscle relaxants, $24(37.5 \%)$ used opioids, and 19

(29.7\%) used nerve pain medications and antidepressants. Other supplemental medication used by some participants included cannabinoids $(21.9 \%)$, steroids $(20.3 \%)$, alcohol (15.6\%), and others (7.8\%) (Fig. 3).

\section{Health Status and Disease Activity}

The mean PROMIS pain interference (66.0 vs. 62.7; $p=0.022)$ and sleep disturbance (61.2 vs. $56.3 ; p=0.017$ ) scores were significantly greater in participants who perceived wear-off versus those who did not $(n=19)$. Physical function scores were significantly lower in participants who perceived a wear-off versus those who did not (36.1 vs. 39.2; $p=0.029$ ) (Fig. 4). In patients experiencing versus not experiencing wear-off, the mean (SD) scores for Depression (2.6 vs. 2.9; $p=0.191$ ) and Global Health (2.0 vs. $2.5 ; p=0.065)$ were not statistically significant. The mean (SD) BASDAI score was significantly greater in participants who perceived wear-off versus those who did not (6.8 vs. $5.3 ; p=0.001)$ (Fig. 4 ). 


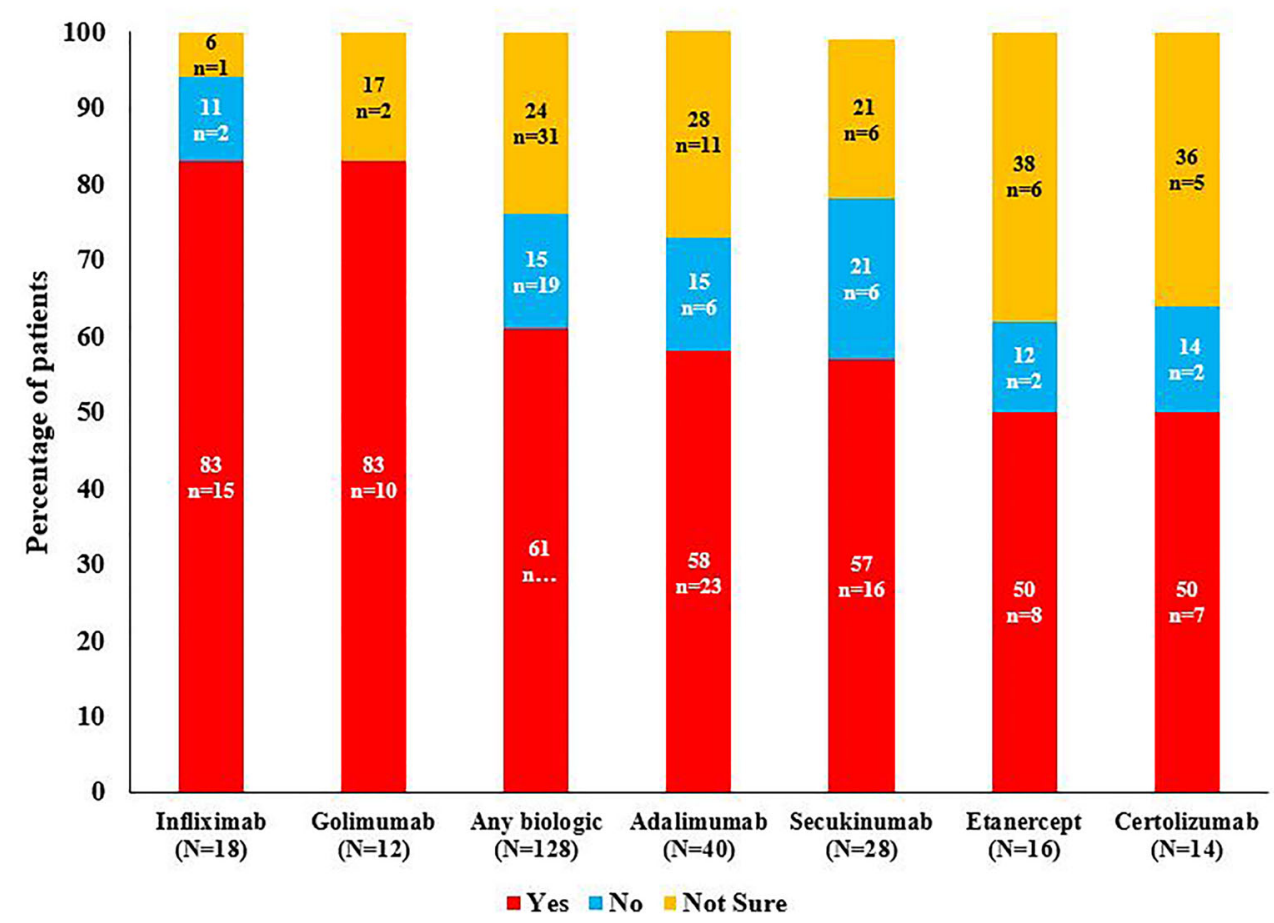

Fig. 2 Percentage of patients reporting that their current biologic wears off before the next dose

\section{DISCUSSION}

This is a real-world, cross-sectional, surveybased study in a predominantly female population. The mean age of patients with axSpA/AS in our study (reporting wear-off and no wear-off between bDMARD doses) was 46 and 48 years, respectively, with most patients reporting high disease activity across all subgroups, which is similar to other existing reports $[22,23]$. We found that most patients experienced treatment satisfaction with bDMARDs, despite high disease activity. In addition, the majority of patients perceived wear-off between doses of their bDMARD treatment and opted to use supplemental medications, predominantly NSAIDs and muscle relaxants, to manage their symptoms during wear-off. We found that variables for improvement of axSpA symptoms, pre-adult onset of axSpA symptoms, lack of positive HLA-B27, and being female were positively associated with dose wear-off. However, wear-off may not be possible in patients who truly do not experience any improvement in
axSpA symptoms, but patients' interpretation of symptom improvement may be variable.

Real-world data on patient treatment satisfaction in axSpA/AS are limited, and very little is known about the influence of biologic wear-off on patient satisfaction. Results from a multicenter observational study in an Italian cohort suggested that patients with axSpA/AS reported higher treatment satisfaction versus patients with nr-axSpA with bDMARDs [24]. A previous online survey-based study showed that, overall, patients with axSpA/AS were satisfied with their rheumatology care; however, unmet needs were also reported, despite $43 \%$ of patients reporting use of biologics [25]. These findings suggest that many patients have favorable experiences with bDMARDs, but bDMARDs may be insufficient for a sizable percentage of axSpA/AS patients. Our study findings suggest that wear-off between doses substantially impairs satisfaction.

Results from another cross-sectional study by the European Map of axSpA/AS mentioned that understanding the patient's perspective can 


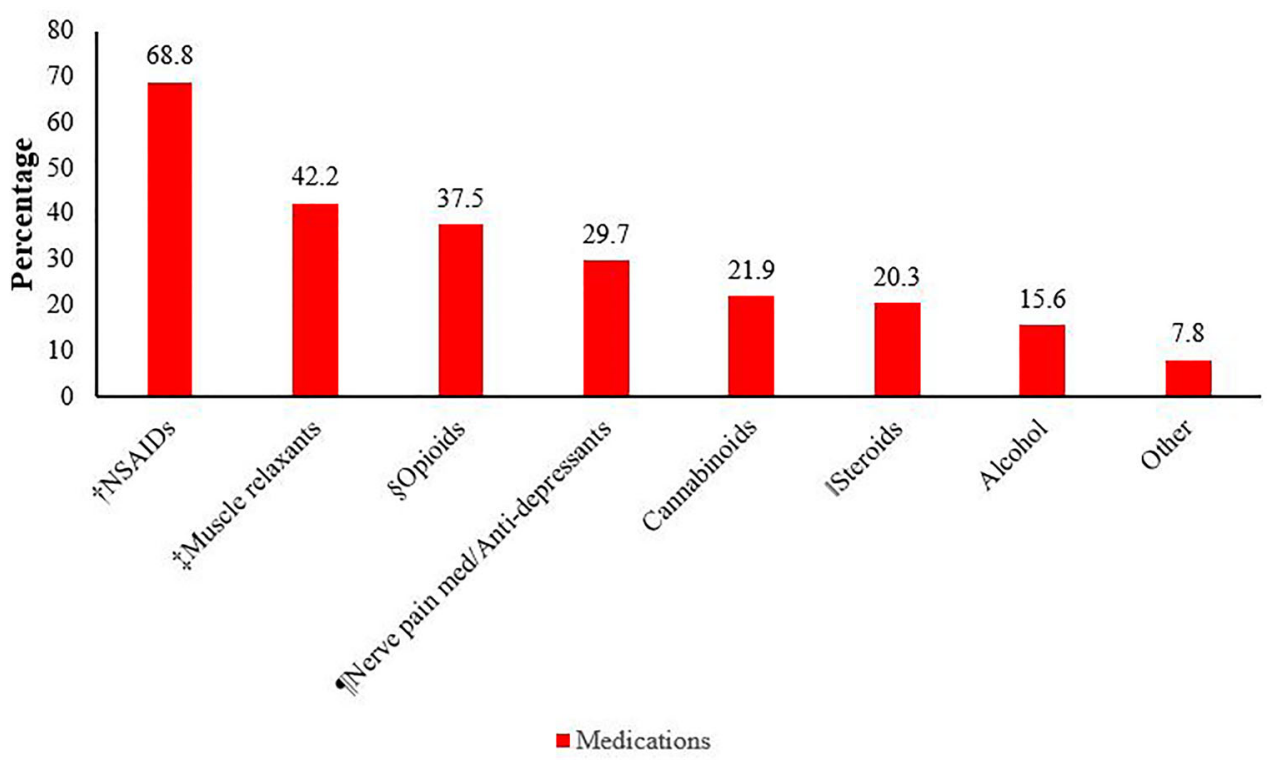

Fig. 3 Additional medications or supplements used when bDMARD wear-off is perceived $(N=64)$. 'Ibuprofen/ $\operatorname{Advil}^{\circledR} /{ }_{\text {Motrin }}{ }^{\circledR}$, naproxen/Aleve ${ }^{\circledR}$, celecoxib/Celebrex ${ }^{\circledR}$, meloxicam/Mobic ${ }^{\circledR}$, diclofenac/Voltaren ${ }^{\circledR}$, etc. ${ }^{\ddagger}$ Carisoprodol/Soma ${ }^{\circledR}$, chlorzoxazone/Parafon Forte ${ }^{\circledR} /$ Lorzone $^{\circledR}$, cyclobenzaprine $/$ Fexmid $^{\circledR} /$ Flexeril $^{\circledR} /$ Amrix ${ }^{\circledR}$, metaxalone/

improve both health outcomes and shared decision-making between patients and rheumatologists [26]. Although many studies report a loss of efficacy or response with bDMARDs [27], to our knowledge, none of the studies discuss the waning efficacy between biologic doses as perceived by patients with axSpA/AS. This study demonstrates that wearoff between doses is common, and that patients experiencing wear-off frequently used supplemental medications to manage symptoms during wear-off. The association of wear-off with unfavorable patient outcomes (i.e., sleep disturbance, increased disease activity) suggests that wear-off negatively impacts patients' lives.

This study has several strengths. To our knowledge, it is the first real-world study in patients with axSpA/AS from the USA to have quantified wear-off of biologic efficacy between doses as one of the plausible reasons for changing axSpA/AS treatment or using supplemental medications to manage the signs and symptoms. This study adds important insight
Skelaxin ${ }^{\circledR} /$ Metaxall $^{\circledR}$, etc. ${ }^{\S}$ Oxycodone/OxyContin ${ }^{\circledR}$, hydrocodone/Vicodin ${ }^{\circledR}$, codeine, morphine. "Gabapentin, pregabalin/Lyrica ${ }^{\circledR}$, duloxetine/Cymbalta ${ }^{\circledR}$. IPrednisone, methylprednisolone betamethasone, hydrocortisone, etc. LDMARD biological disease-modifying antirheumatic drugs, NSAID non-steroidal anti-inflammatory drugs

into the perspective and experience of patients regarding axSpA/AS treatment, especially women living with axSpA/AS and experiencing high disease activity.

The findings of our study must be interpreted in the context of some limitations of a survey-based design. Since this is a cross-sectional study, it is challenging to completely establish any cause-effect relationship. Patient perspectives may be subject to patients' bias and experience. Participants in the study are part of an online registry and patient community; they may be more likely to take part regularly in research studies, and thus may have had a greater interest in managing their disease. There is also potential for selection bias. Although axSpA/AS is typically considered to be a maledominant disease [28], $82.8 \%$ of our study was female, perhaps indicating that women are typically more active online for such topics. Another contributing factor to a higher female population in our study is patient recruitment through the ArthritisPower registry, in which 

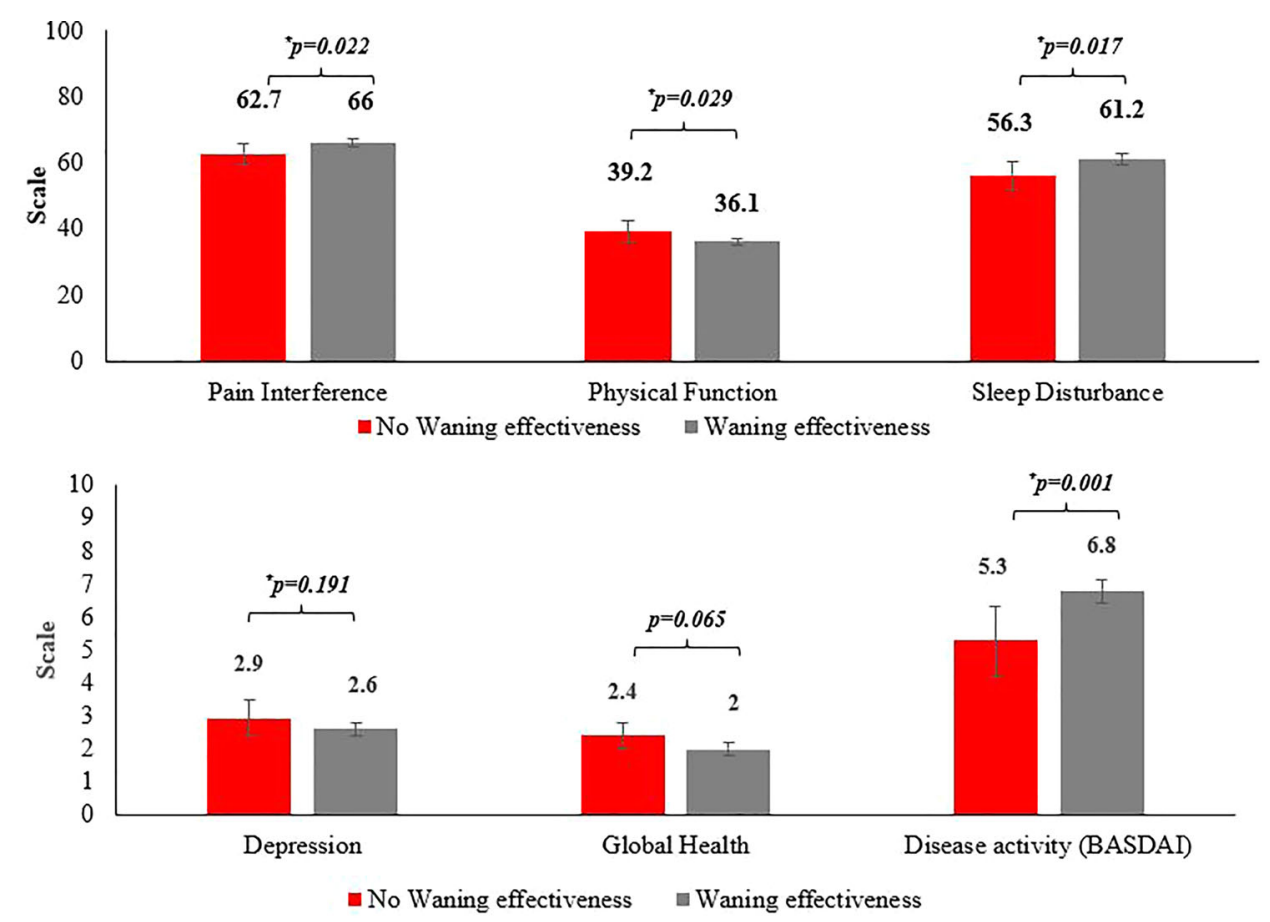

Fig. 4 Health status and disease activity scores in patients with and without waning effectiveness between biologic doses. $n$ for Waning Effectiveness is 78 and the $n$ for no waning effectiveness is 19 (excludes unsure). *Statistical significance between groups of patients who experienced wear-off between bDMARD doses and those who

$88 \%$ of the population is female. Therefore, this study population is not representative of the general patient population with axSpA/AS. The study relied on patients' self-report of physician diagnosis of axSpA/AS, which may have under- or overrepresented symptoms, and provider confirmation of diagnosis was not obtained.

\section{CONCLUSIONS}

In summary, the majority of bDMARD-treated patients with axSpA/AS expressed satisfaction with treatment, despite relatively high disease activity. However, most patients experienced wear-off between doses and used supplemental medications to manage the symptoms. This study may be used to inform future efforts to better understand the impact of wear-off on experienced no wear-off or were not sure, $p<0.05$. For depression and global health, higher scores indicate greater severity. BASDAI is scored on a $0-10$ scale with score $\geq 4$ indicating suboptimal control of disease. BASDAI Bath Ankylosing Spondylitis Disease Activity Index, CI 95\% confidence interval, $S D$ standard deviation

patients' lives and to develop strategies for addressing wear-off.

\section{ACKNOWLEDGEMENTS}

The authors would like to thank Jennifer Walker, who was a part of the early study design discussions from the perspective of patients with axial spondyloarthritis.

Thanking Participants. The authors would like to thank all the patients in ArthritisPower registry who participated in the survey program.

Funding. The study and journal's Rapid Service Fee were funded by Eli Lilly and Company, USA. 
Authorship. All named authors meet the International Committee of Medical Journal Editors (ICMJE) criteria for authorship, take responsibility for the integrity of the work as a whole, and have given their approval for this version to be published.

Author Contributions. William Benjamin Nowell contributed to the conception and design of the study and acquisition/analysis/ interpretation of data. Jeffrey $\mathrm{R}$ Lisse contributed to interpretation of the data and critical revision of the work for important intellectual content. Theresa Hunter, Jeffrey R Curtis, and Jessica Walsh contributed to conception and design of the study, interpretation of the data, and critical revision of the work for important intellectual content. Carol Himelein contributed to the design of the study, interpretation of the data, and critical revision of the work for important intellectual content. Kelly Gavigan and Rebecca J Bolce contributed to acquisition and analysis of the data and critical revision of the work for important intellectual content. Suchita Dubey contributed to interpretation of the data and drafting of the work.

Prior Presentation. Some of these data were presented at the European Alliance of Associations for Rheumatology formerly the European League Against Rheumatism 2021. The abstract from this data is published in 'Annals of Rheumatic Diseases' and citable as Nowell WB et al. POS1499- PARE Patient perspectives of biologic treatments for axial spondyloarthritis: satisfaction, wear-off between doses, and use of supplemental medications. Annals of the Rheumatic Diseases 2021;80:1034-1035.

Disclosures. Theresa Hunter, Carol Himelein, Jeffrey R Lisse, and Rebecca J Bolce are employees and stockholders of Eli Lilly and Company. Suchita Dubey is an employee of Eli Lilly and Company. Jessica Walsh received grants or contracts from Pfizer, AbbVie, and Merck and consulting fees from Janssen, Eli Lilly, Novartis, Amgen, UCB, AbbVie, and
Pfizer. Jeffery Curtis received grants or contracts or consulting fees from AbbVie, Amgen, BMS, CorEvitas, Janssen, Lilly, Novartis, Myriad, Pfizer, Sanofi, Setpoint, Scipher, and UCB. Kelly Gavigan and William Benjamin Nowell are employees of the Global Healthy Living Foundation (GHLF). GHLF receives grants, sponsorships, and contracts from pharmaceutical manufacturers and private foundations. A full list of GHLF funders is publicly available here: https://www.ghlf.org/our-partners/. William Benjamin Nowell was principal investigator for research funded by Eli Lilly and Company, AbbVie, and PICORI and payments were made to Global Healthy Living Foundation.

Compliance with Ethics Guidelines. The study was reviewed and deemed exempt by Advarra Institutional Review Board (Advarra IRB protocol \#00039559).

Data Availability. The datasets analyzed during the current study are from the ArthritisPower Registry and can be made available by requesting from the corresponding author on a reasonable request.

Open Access. This article is licensed under a Creative Commons Attribution-NonCommercial 4.0 International License, which permits any non-commercial use, sharing, adaptation, distribution and reproduction in any medium or format, as long as you give appropriate credit to the original author(s) and the source, provide a link to the Creative Commons licence, and indicate if changes were made. The images or other third party material in this article are included in the article's Creative Commons licence, unless indicated otherwise in a credit line to the material. If material is not included in the article's Creative Commons licence and your intended use is not permitted by statutory regulation or exceeds the permitted use, you will need to obtain permission directly from the copyright holder. To view a copy of this licence, visit http://creativecommons.org/licenses/by$\mathrm{nc} / 4.0 /$. 


\section{REFERENCES}

1. Rudwaleit M, Metter A, Listing J, Sieper J, Braun J. Inflammatory back pain in ankylosing spondylitis: a reassessment of the clinical history for application as classification and diagnostic criteria. Arthritis Rheum. 2006;54(2):569-78.

2. Sieper J, van der Heijde D, Landewé R, Brandt J, Burgos-Vagas R, Collantes-Estevez E, et al. New criteria for inflammatory back pain in patients with chronic back pain: a real patient exercise by experts from the Assessment of SpondyloArthritis international Society (ASAS). Ann Rheum Dis. 2009;68(6): 784-8.

3. Strand V, Singh JA. Evaluation and management of the patient with suspected inflammatory spine disease. Mayo Clin Proc. 2017;92(4):555-64.

4. Reveille JD, Witter JP, Weisman MH. Prevalence of axial spondylarthritis in the United States: estimates from a cross-sectional survey. Arthritis Care Res (Hoboken). 2012;64(6):905-10.

5. Ward MM, Deodhar A, Gensler LS, Dubreuil M, Yu D, Khan MA, et al. 2019 Update of the American College of Rheumatology/Spondylitis Association of America/spondyloarthritis research and treatment network recommendations for the treatment of ankylosing spondylitis and nonradiographic axial spondyloarthritis. Arthritis Care Res (Hoboken). 2019;71(10):1285-99.

6. Sieper J, Braun J, Rudwaleit M, Boonen A, Zink A. Ankylosing spondylitis: an overview. Ann Rheum Dis. 2002;61(suppl 3):iii8.

7. Packham J. Optimizing outcomes for ankylosing spondylitis and axial spondyloarthritis patients: a holistic approach to care. Rheumatology. 2018;57(suppl_6):vi29-33.

8. Davis JC Jr, Van Der Heijde D, Braun J, Dougados M, Cush J, Clegg DO, et al. Recombinant human tumor necrosis factor receptor (etanercept) for treating ankylosing spondylitis: a randomized, controlled trial. Arthritis Rheum. 2003;48(11):3230-6.

9. Dougados M, Braun J, Szanto S, Combe B, Elbaz M, Geher P, et al. Efficacy of etanercept on rheumatic signs and pulmonary function tests in advanced ankylosing spondylitis: results of a randomised double-blind placebo-controlled study (SPINE). Ann Rheum Dis. 2011;70(5):799-804.

10. Dougados M, van der Heijde D, Sieper J, Braun J, Maksymowych WP, Citera G, et al. Symptomatic efficacy of etanercept and its effects on objective signs of inflammation in early nonradiographic axial spondyloarthritis: a multicenter, randomized, double-blind, placebo-controlled trial. Arthritis Rheumatol. 2014;66(8):2091-102.

11. van der Heijde D, Dijkmans B, Geusens P, Sieper J, DeWoody K, Williamson P, et al. Efficacy and safety of infliximab in patients with ankylosing spondylitis: results of a randomized, placebo-controlled trial (ASSERT). Arthritis Rheum. 2005;52(2): 582-91.

12. van der Heijde D, Kivitz A, Schiff MH, Sieper J, Dijkmans BA, Braun J, et al. Efficacy and safety of adalimumab in patients with ankylosing spondylitis: results of a multicenter, randomized, doubleblind, placebo-controlled trial. Arthritis Rheum. 2006;54(7):2136-46.

13. Smolen JS, Braun J, Dougados M, Emery P, Fitzgerald $\mathrm{O}$, Helliwell $\mathrm{P}$, et al. Treating spondyloarthritis, including ankylosing spondylitis and psoriatic arthritis, to target: recommendations of an international task force. Ann Rheum Dis. 2014;73(1): 6-16.

14. Uhrenholt L, Schlemmer A, Hauge E-M, Christensen R, Dreyer L, Suarez-Almazor ME, et al. Dosage reduction and discontinuation of biological disease-modifying antirheumatic drugs in patients with rheumatoid arthritis, psoriatic arthritis and axial spondyloarthritis: protocol for a pragmatic, randomised controlled trial (the BIOlogical Dose OPTimisation (BIODOPT) trial). BMJ Open. 2019;9(7):e028517-e.

15. Nowell WB, Gavigan $\mathrm{K}$, Hunter T, Malatestinic WN, Bolce RJ, Lisse JR, Himelein C, Curtis JR, Walsh JA. Treatment satisfaction and decision-making from the patient perspective in axial spondyloarthritis: real-world data from a descriptive cross-sectional survey study from the arthritispower registry. ACR Open Rheumatol. 2021. https://doi.org/10.1002/ acr2.11365.

16. Rojavin MA, Hubsch A, Lawo JP. Quantitative evidence of wear-off effect at the end of the intravenous IgG (IVIG) dosing cycle in primary immunodeficiency. J Clin Immunol. 2016;36(3): 210-9.

17. Nowell WB, Curtis D, Thai M, Wiedmeyer C, Gavigan K, Venkatachalam S, et al. Digital interventions to build a patient registry for rheumatology research. Rheum Dis Clin N Am. 2019;45(2): 173-86.

18. Nowell WB, Curtis JR, Crow-Hercher R. Patient governance in a patient-powered research network for adult rheumatologic conditions. Med Care. 2018;56(10 Suppl):S16-21.

19. Garrett S, Jenkinson T, Kennedy LG, Whitelock H, Gaisford P, Calin A. A new approach to defining 
disease status in ankylosing spondylitis: the Bath Ankylosing Spondylitis Disease Activity Index. J Rheumatol. 1994;21(12):2286-91.

20. Health NIo. Patient-Reported Outcomes Measurement Information System (PROMIS $\left.{ }^{\circledR}\right) .2021$. https://www.healthmeasures.net/.

21. Hwang MC, Ogdie A, Puravath A, Reveille JD. Reliability and validity of patient-reported outcomes measurement information system short forms in ankylosing spondylitis. J Rheumatol. 2020;47(8):1182-8.

22. Law L, Beckman Rehnman J, Deminger A, Klingberg E, Jacobsson LTH, Forsblad-d'Elia H. Factors related to health-related quality of life in ankylosing spondylitis, overall and stratified by sex. Arthritis Res Ther. 2018;20(1):284.

23. Ogdie A, Benjamin Nowell W, Reynolds R, Gavigan K, Venkatachalam S, de la Cruz M, et al. Real-world patient experience on the path to diagnosis of ankylosing spondylitis. Rheumatol Ther. 2019;6(2): 255-67.

24. D'Angelo S, Gilio M, D'Attino RM, Gualberti G, Merolla R, di Luzio PU, et al. Observational study on the QUality of life of Italian Axial SpondyloARthritis patients (QUASAR): baseline data. Clin Exp Rheumatol. 2019;37(5):748-55.

25. Derakhshan $\mathrm{MH}$, Pathak H, Cook D, Dickinson S, Siebert S, Gaffney K, et al. Services for spondyloarthritis: a survey of patients and rheumatologists. Rheumatology. 2018;57(6):987-96.

26. Garrido-Cumbrera M, Poddubnyy D, Gossec L, Gálvez-Ruiz D, Bundy C, Mahapatra R, et al. The European map of axial spondyloarthritis: capturing the patient perspective-an analysis of 2846 patients across 13 countries. Curr Rheumatol Rep. 2019;21(5):19.

27. Deodhar A, Sandoval D, Holdsworth E, Booth N, Hunter T. Use and switching of biologic therapy in patients with non-radiographic axial spondyloarthritis: a patient and provider survey in the United States. Rheumatol Ther. 2020;7(2):415-23.

28. Rusman T, van Vollenhoven RF, van der HorstBruinsma IE. Gender differences in axial spondyloarthritis: women are not so lucky. Curr Rheumatol Rep. 2018;20(6):35. 